\title{
If horses entrain, don't entirely reject vocal learning: An experience-based vocal learning hypothesis
}

\author{
ADENA SCHACHNER \\ Department of Psychology, Harvard University
}

\begin{abstract}
Bregman and colleagues describe methods for testing whether horses entrain their actions to an auditory beat. If horses can entrain, does this necessarily imply that there is no causal relationship between vocal learning and entrainment? I propose an alternative way in which vocal learning may relate to entrainment - one that is consistent with entrainment in some vocal non-learning species. Due to engaging in the developmental process of vocal learning, there may be early experiences common to vocal learners, but rare in vocal non-learning species. It is possible that it is these experiences that are critical for entrainment - not vocal learning itself, nor related genes. These experiences may result in critical changes in neural development, leading to the development of cognitive mechanisms necessary for both vocal learning and entrainment. This hypothesis changes the causal story from one of genetic change to one of changes in experience, and from a focus on evolution to a focus on individual ontogeny. Thus, if horses can entrain, we should not immediately reject the idea of a relationship between vocal learning and entrainment: First, we should consider whether some unusual aspect of the horses' experience effectively replicates the unusual experiences of vocal learning animals.
\end{abstract}

Submitted 2013 Jan 11; accepted 2013 Jan 14.

KEYWORDS: entrainment, vocal learning, vocal imitation, evolution, development

THE vocal learning hypothesis predicts that only vocal mimicking animals should be able to entrain, or synchronize their movements with an auditory beat. Thus far, comparative evidence involving a number of species has borne out this prediction (Patel, Iversen, Bregman \& Schulz, 2009; Schachner, Brady, Pepperberg \& Hauser, 2009). In the current paper, however, Bregman and colleagues describe anecdotal reports suggesting that horses may be able to entrain as well, in spite of their lack of vocal learning ability. This observation is important to test empirically: As Bregman notes, evidence of entrainment in horses would refute the vocal learning hypothesis. To this end, Bregman and colleagues describe methods for testing whether horses are able to entrain their actions to an auditory beat. By laying out necessary information in a clear step-by-step manner, Bregman and colleagues make it possible for other authors with access to horses to easily test for entrainment.

Individual animals within a species may differ in their tendency to entrain, and even a single animal may not respond to all rhythmic stimuli or may at times fail to move in response to music (Patel et al., 2009; Schachner et al., 2009). Thus, to have reasonable power to detect entrainment in a species, it is necessary to obtain data from a large range of subjects, in a variety of different situations, with a variety of different stimuli. By allowing for data collection from a larger range of subjects, the current paper serves a valuable purpose.

In addition, the current paper points out the value of testing trained animals. Such animals may be instructed to produce an isochronous, repetitive movement (such as walking), which may then potentially become entrained. This method avoids a major risk of comparative entrainment research: the risk that the subject fails to produce any movement whatsoever. If a subject remains still during entrainment testing, this provides a null result, but also a complete lack of relevant data - thus, the null result is difficult to interpret. In selecting new animals and new species to test for entrainment, it may be useful to begin by testing animals which can be instructed to produce an isochronous, repetitive movement - one which has a wide range of possible tempos, and thus may potentially become entrained. 


\section{IF HORSES ENTRAIN: AN ALTERNATIVE TO THE VOCAL LEARNING HYPOTHESIS}

If we find that horses can entrain, how might we make sense of the overall pattern of comparative data? Would this finding necessarily imply that there is no causal relationship between vocal learning and entrainment? I agree with Bregman and colleagues that such a finding would refute the current vocal learning hypothesis. However, I believe that even in this case, it is possible that vocal learning may play a role. Here I propose an alternative way in which vocal learning may relate to entrainment - one that is consistent with entrainment in some vocal non-learning species.

In the original vocal learning hypothesis, Patel hypothesized that natural selection for vocal learning gave us the required cognitive machinery for entrainment (Patel, 2006; Patel et al., 2009). This hypothesis implies that the crucial changes were genetic in nature: certain genes emerged and were favored through natural selection, and these genes were needed for the development of neural mechanisms used for vocal learning and entrainment. Without these genes, the required brain mechanisms cannot develop, and both vocal learning and entrainment are impossible. By this hypothesis, other factors, like having certain experiences, should not be sufficient to allow vocal non-learning species to entrain.

However, there is an alternative way in which vocal learning may play a role: by shaping experience. Due to engaging in the developmental process of vocal learning, there may be early experiences that are common to all vocal learners, but rare in vocal non-learning species. For instance, vocal learning birds and humans go through analogous stages of vocalization development (Doupe \& Kuhl, 1999). During vocal learning, individuals develop fine motor control (over the vocal apparatus) and hone their motor programs by comparing the sound of their own vocalizations to an auditory template stored in memory, and engaging in error correction based on this real-time feedback (Konishi, 1965; Doupe \& Kuhl, 1999).

If vocal learning causes animals to have certain common experiences, it is possible that it is these experiences that are critical for entrainment - not vocal learning itself, nor related genes. These hypothesized necessary experiences may result in critical changes in neural development, leading to the development of cognitive mechanisms necessary for both vocal learning and entrainment. This process would be analogous to that of reading, another capacity with domain-specific neural mechanisms that only develop with specific experience (Dehaene, 2009).

If it is the unusual experiences of vocal learners that are critical for entrainment (instead of specific genetic factors), this leaves open the possibility that the required experiences may be available to some animals that are not vocal learners, through the introduction of unusual experiences. One possibility is that the process by which trained horses (e.g. dressage horses) develop fine motor control, and learn motor skills, may be meaningfully similar to the process of developing fine motor control for vocal learning. For instance, the horses' riders may train them to produce specific movements using real-time feedback and error correction, perhaps engaging in a process similar to that which occurs naturally in vocal learning species.

This alternative hypothesis changes the causal story from one of genetic change to one of change in experience, and from a focus on evolution to a focus on individual ontogeny. However, this hypothesis maintains an important role for vocal learning, positing that vocal learning leads animals to have certain early experiences, which in turn cause them to develop the neural mechanisms necessary for entrainment. Thus, if we do find that horses can entrain, we should not immediately reject the idea of a relationship between vocal learning and entrainment. First we should consider the role of vocal learning in guiding early experience, and ask whether some unusual aspect of the horses' experience effectively replicates the unusual experiences of vocal learning animals.

\section{REFERENCES}

Dehaene, S. (2009). Reading in the Brain: The Science and Evolution of a Human Invention. New York: Viking Press.

Doupe, A.J., \& Kuhl, P.K. (1999). Birdsong and human speech: common themes and mechanisms. Annual Review of Neuroscience, Vol. 22, No.1, pp. 567-631.

Konishi, M. (1965). The role of auditory feedback in the control of vocalization in the whitecrowned sparrow. Zeitschrift für Tierpsychologie, Vol. 22, pp. 770-783. 
Patel, A.D. (2006). Musical rhythm, linguistic rhythm, and human evolution. Music Perception, Vol. 24, No.1, pp. 99-104.

Patel, A.D., Iversen, J.R., Bregman, M.R., \& Schulz, I. (2009). Experimental evidence for synchronization to a musical beat in a nonhuman animal. Current Biology, Vol. 19, No.10, pp. 827-830.

Schachner, A., Brady, T.F., Pepperberg, I.M., \& Hauser, M.D. (2009). Spontaneous motor entrainment to music in multiple vocal mimicking species. Current Biology, Vol.19, No.10, pp. 831-836. 